\section{RSP}

http://www.rsp.fsp.usp.br/
Revista de Saúde Pública

\title{
Associação entre raça/cor da pele e parto prematuro: revisão sistemática com meta-análise
}

Kelly Albuquerque de Oliveira', Edna Maria de Araújo", Keyte Albuquerque de Oliveira"', Cesar Augusto Casotti"v, Carlos Alberto Lima da Silva", Djanilson Barbosa dos Santos ${ }^{\vee}$

' Universidade Estadual de Feira de Santana. Programa de Pós-Graduação em Saúde Coletiva. Feira de Santana, BA, Brasil

" Universidade Estadual de Feira de Santana. Departamento de Saúde. Feira de Santana, BA, Brasil

III Faculdade Nobre. Feira de Santana, BA, Brasil

iv Universidade Estadual do Sudoeste da Bahia. Departamento de Saúde. Jequié, BA, Brasil

$\checkmark$ Universidade Federal do Recôncavo da Bahia. Centro de Ciências da Saúde. Santo Antônio de Jesus, BA, Brasil

\section{RESUMO}

OBJETIVO: Analisar a associação entre raça/cor da pele e a ocorrência da prematuridade.

MÉTODOS: Meta-análise com estudos observacionais, selecionados por revisão sistemática em bases de dados bibliográficos Medline e Biblioteca Virtual da Saúde com os descritores: "Race or ethnic group" e "ethnicity and health" associados às palavras "infant premature" e "obstetric labor premature". Foram incluídos os artigos publicados no período de 2010 a 2014, do tipo epidemiológico observacional, nas línguas portuguesa, inglesa e espanhola. Foram excluídos os artigos que não possuíam resumos ou que fossem artigos de revisão, teses, dissertações e editorias. Foi adotado o risco relativo e seus respectivos intervalos de confiança (IC95\%) como medidas de efeito, obtidos por meio do modelo de efeito aleatório e representados a partir do gráfico do tipo forest plot. Para analisar os possíveis vieses de publicação e qualidade dos estudos, foi utilizado o teste de Egger e a escala de Newcastle-Ottawa, respectivamente.

Kelly Albuquerque de Oliveira Rua Wanderley de Carvalho, 60 Casa E16 Cond. Viva Mais 1 Pedra do Descanso

44007-204 Feira de Santana, BA, Brasil

E-mail: kellyalbuquerque84@gmail.com

Recebido: 9 dez 2016

Aprovado: 10 abr 2017

Como citar: Oliveira KA, Araújo EM, Oliveira KA, Casotti CA Silva CAL, Santos DB. Associação entre raça/cor da pele e parto prematuro: revisão sistemática com meta-análise. Rev Saude Publica. 2018;52:26.

Copyright: Este é um artigo de acesso aberto distribuído sob os termos da Licença de Atribuição Creative Commons, que permite uso irrestrito, distribuição e reprodução em qualquer meio, desde que o autor e a fonte

originais sejam creditados.

RESULTADOS: Dos 926 artigos identificados, 17 foram elegíveis para o estudo. Dos 17 textos completos publicados, sete eram estudos de coorte retrospectiva, nove eram transversais e um era caso-controle. Com exceção de um estudo, os demais relataram associação positiva entre a raça/cor da pele e a prematuridade. Comparado com recém-nascidos a termo, o risco relativo do efeito combinado naqueles que nasceram prematuros foi de 1,51 (IC95\% 1,39-1,69). O gráfico de funil sugeriu viés de publicação.

CONCLUSÕES: A presente meta-análise indicou uma associação positiva para o risco da prematuridade segundo a raça/cor da pele.

DESCRITORES: Nascimento Prematuro, epidemiologia. Origem Étnica e Saúde. Metanálise. 


\section{INTRODUÇÃO}

A prematuridade (PMT) é um indicador importante para a saúde infantil, uma vez que ela aumenta o risco de ocorrência de doenças crônicas ${ }^{1}$. Representa a maior causa de morbimortalidade neonatal no mundo e gera custos altíssimos para os países ${ }^{2}$.

Uma revisão sistemática sobre a incidência da prematuridade no mundo estimou que 12,9 milhões de nascimentos eram prematuros, cerca de 9,6\% de todos os nascimentos em todo o mundo. Destes, 85,0\% estavam concentrados na África e Ásia, com 10,9 milhões de nascimentos prematuros, 0,5 milhões na Europa e na América do Norte e 0,9 milhões na América Latina e no Caribe 3 .

A prevalência de nascimentos pré-termo nos Estados Unidos aumentou de 9,5\%, em 1981, para $12,7 \%$, em 2005, mantendo-se atualmente na faixa de 12,0\% a 13,0\%, enquanto na Europa esses valores variam entre 5,0\% e 9,0\% ${ }^{4}$. No Brasil, foi possível observar a tendência temporal do crescimento nas taxas da prematuridade. A análise de dados do Sinasc mostrou que a prevalência foi de 5,0\% em 1994, 5,4\% em 1998, 5,6\% em 2000 e 6,5\% em 20045; a prevalência foi de 6,0\% e 7,0\%, de 2000 a 2010, com dados do Sinasc. Os valores ficaram entre 11,0\% e $12,0 \%$ para o mesmo período após correção e 11,7\% a 11,8\%, no triênio 2009-2011 .

A desigualdade socioeconômica e étnico-racial é documentada como risco para a ocorrência da $\mathrm{PMT}^{7}$. No que se refere à desigualdade étnico-racial, como ocorre, por exemplo, nos Estados Unidos, a raça/cor tem sido evidenciada como um importante determinante social para a saúde da população, pois o tratamento da variável raça/cor da pele apenas como variação genética não explica os diferenciais em saúde por diferentes grupos de $\operatorname{cor}^{8-11}$.

Nesse sentido, é importante elucidar a teoria de determinação social, em que a posição social ocupada pelo indivíduo contribui de maneira significativa para a ocorrência das doenças e também para sua distribuição desigual ${ }^{12-15}$.

Dessa forma, a variável raça/cor precisa ser discutida como determinante social na ocorrência das doenças e agravos à saúde e não como mero determinismo biológico, uma vez que a raça/cor carrega um arcabouço histórico-social nos diferenciais de grupo.

Estudos mostram que as taxas de incidência de partos prematuros são desiguais segundo a raça/cor da pele. Mulheres negras têm o risco 2,5 vezes maior de ocorrência de parto pré-termo em comparação com mulheres brancas, e essas diferenças raciais têm aumentado desde 1990 $0^{7,16,17}$.

Há evidências de que as disparidades étnico-raciais podem levar à prematuridade. Estudo realizado nos Estados Unidos observou variação considerável nos resultados dos nascimentos segundo a raça/cor da pele materna: $18,4 \%$ dos partos de mulheres negras ocorreram antes de 37 semanas de gestação ${ }^{18}$. Estudo de coorte no Reino Unido com o objetivo de comparar o tempo de duração da gestação entre as mulheres brancas e as mulheres negras concluiu que estas têm 1,5 vezes mais chances de ter partos prematuros ${ }^{19}$.

No Brasil, foi observada uma persistente situação desfavorável para as mulheres de cor preta e parda. Elas apresentaram menores chances de passar por consultas ginecológicas e de pré-natal e chances ainda menores de realizar a primeira consulta de pré-natal em período igual ou inferior ao quarto mês de gravidez ${ }^{6,20}$. Dados de uma coorte de Ribeirão Preto, SP, mostrou que a cor da pele é um fator de risco independente para a PMT, mesmo após ajuste da renda familiar e escolaridade materna. Isso sugere que as diferenças raciais em relação à PMT são explicadas pela desvantagem socioeconômica vivida pelas mulheres negras, mas também são influenciadas por outros fatores, possivelmente por discriminação racial ${ }^{21}$.

A análise dos resultados dos partos por diferenças étnicas ou raciais maternas faz-se necessária, pois podem sugerir hipóteses etiológicas, destacar as implicações nos desfechos gestacionais e direcionar para uma melhoria da qualidade das consultas de pré-natal ${ }^{22,23}$.Embora tenham sido publicadas várias revisões sistemáticas sobre a associação entre alguns dos aspectos da raça/cor 
da pele em relação à $\mathrm{PMT}^{7,16}$, os autores do presente estudo identificaram uma meta-análise apenas relacionando a ocorrência da PMT diretamente com a raça/cor da pele. Selecionaram artigos de 1983 a 2011 e trabalharam com medidas de associação ajustadas ${ }^{24}$. Assim, para agregar evidências e sistematizar as informações sobre a associação entre a raça/cor da pele e a PMT, esse estudo foi conduzido com o propósito de fornecer informações para subsidiar o planejamento de futuros estudos e políticas públicas para a prevenção do parto prematuro e suas consequências para a qualidade de vida da população e redução de custos para os serviços de saúde.

A hipótese deste estudo é que as mulheres negras têm um risco maior de ter partos prematuros quando comparadas com as mulheres não negras. O objetivo do presente estudo foi realizar uma meta-análise para analisar a associação entre a raça/cor da pele das gestantes e a ocorrência da PMT.

\section{MÉTODOS}

Revisão sistemática com meta-análise, com base na declaração Preferred Reporting Items for Systematic Reviews and Meta-Analyses (PRISMA), a qual é composta por 27 itens que auxiliam na elaboração, análise, publicação de meta-análises e revisões sistemáticas de estudos observacionais ${ }^{25}$. Foi elaborado um protocolo de pesquisa para auxiliar na busca, extração dos dados, análise e interpretação dos artigos.

Foram incluídos os artigos com desenho epidemiológico observacional analítico que respondessem aos critérios: ser original com resumo disponível, ter sido publicado nos últimos cinco anos (2010 a 2014), ter sido publicado em língua portuguesa, inglesa e espanhola, e que apresentassem somente dados primários para o cálculo da medida de associação entre a raça/cor da pele e a prematuridade. Foram excluídos os artigos sem resumos e que fossem artigos de revisão, teses/dissertações e editorias.

Para a busca dos artigos, utilizou-se o sistema integrado de busca da Biblioteca Virtual em Saúde Brasil (BVS) e do Medline e PubMed, bases de dados que reúnem as publicações de periódicos científicos na área de saúde. Visou-se encontrar publicações de artigos científicos relacionados à associação entre a raça/cor da pele e a prematuridade gestacional.

Utilizou-se o método de pesquisa integrado para as buscas em todos os índices e todas as fontes. Isso permitiu ampla busca, integrando várias bases de dados e uma busca detalhada, por relevância de títulos, resumos e textos. As palavras usadas para a busca foram: "Race or ethnic group" e "ethnicity and health" associadas às palavras "infant premature" e "obstetric labor premature".

Ao se fazer a pesquisa utilizando-se a associação dos descritores, na BVS de outubro e novembro de 2015, foram encontrados 1.163 resultados de textos, dos quais 372 eram textos publicados a partir de 2010. A busca no Medline e Pubmed utilizando a mesma associação de descritores encontrou 1.907 resultados, 554 publicados a partir de 2010.

Participaram do estudo as mulheres gestantes que tiveram registro da idade gestacional no nascimento do recém-nascido. O desfecho foi a PMT, referenciado como a ocorrência do nascimento antes da $37^{\mathrm{a}}$ semana de gestação. A variável de exposição foi a raça/cor da pele. Todas as participantes de raça/cor da pele negra, que corresponderam às mulheres pretas e pardas, foram consideradas grupo de exposição e as mulheres de raça/cor da pele branca foram consideradas grupo de referência.

Dois revisores analisaram de forma independente todo o processo de seleção dos estudos, analisando os títulos e os resumos. Os artigos foram lidos na íntegra, seguindo os critérios de inclusão e exclusão estabelecidos.

Os dados de cada artigo elegível para o estudo foram extraídos e organizados em uma tabela resumo que continha os seguintes pontos: autores e data de publicação, delineamento do estudo, e as medidas de associação. Os artigos foram avaliados quanto à qualidade metodológica por meio do instrumento Newcastle-Ottawa Scale (NOS), que avalia a qualidade 
dos estudos de coorte, transversal e caso-controle para detecção de vieses ${ }^{17}$. Os estudos com baixa qualidade metodológica foram excluídos. A qualidade dos estudos foi baseada na classificação dos artigos nas seguintes categorias: ruim, regular, bom e excelente.

Os estudos foram analisados quanto à qualidade metodológica nos seguintes quesitos: quanto à seleção dos estudos, na qual foi observada a representatividade da amostra; quanto à exposição e o desfecho de interesse; quanto ao poder de comparação dos achados; e quanto ao efeito através da avaliação dos resultados. Obtivemos, assim, a seguinte classificação dos artigos: três regulares, 10 bons e quatro excelentes.

Os dados coletados foram expressos em números absolutos e inseridos em tabelas de contingências (2x2) para o cálculo da medida de associação. Foram selecionados estudos que relataram somente dados primários. Foram realizadas as análises descritiva e meta-analítica dos dados. O risco relativo (RR) e o intervalo de $95 \%$ de confiança foram utilizados como medidas de efeito.

Para verificar a heterogeneidade e a consistência dos estudos, foi utilizado o teste Q de Cochran e I ${ }^{2}$. Empregou-se o modelo aleatório e o modelo de regressão de Egger, gerando o gráfico forest plot.

Os resultados da ocorrência da PMT associada à raça/cor da pele foram meta-analisados por meio do programa estatístico Stata.

Após pesquisa na BVS utilizando-se a associação dos descritores, foram encontrados 1.163 resultados de textos, dos quais 372 foram publicados a partir de 2010. A busca no MedLine e PubMed utilizando a mesma associação de palavras encontrou 1.907 resultados, 554 publicados a partir de 2010 (Tabela 1).

Foram identificados 3.070 registros nas bases de dados, dos quais 926 artigos se referiam ao período de 2010 a 2014. Desse total, foram excluídos 83 artigos por duplicação (Figura 1). Foi realizada uma leitura exploratória com o objetivo de obter uma visão geral dos 926 artigos, com a leitura dos títulos e resumos para verificar quais seriam incluídos no estudo.

Foram excluídos 843 artigos por não atenderem aos critérios de inclusão, resultando em 99 artigos para a leitura na íntegra. Após a análise com base nos critérios de elegibilidade, 17 artigos foram incluídos; foi realizada uma leitura minuciosa e a extração dos dados. Os 17 textos completos publicados adotaram como critério para determinar a raça/cor, a autoclassificação, baseada na cor e nas características físicas das participantes. Desses, sete se tratavam de estudos de coorte retrospectiva, nove de estudos transversais e um de estudo caso-controle.

As razões para a exclusão dos artigos foram: disparidade étnico-racial baseada na área geográfica $(n=14)$, delineamento metodológico fraco $(n=14)$, ensaio teórico $(n=5)$, interação com complicações gestacionais ou de saúde $(\mathrm{n}=6)$, disparidade étnico-racial baseada na migração $(n=19)$, disparidade étnico-racial baseada nos serviços de saúde ofertados $(n=14)$, e disparidade étnico-racial baseada em marcadores genéticos $(\mathrm{n}=10)$.

Tabela 1. Distribuição dos resultados da busca dos artigos sobre raça/cor da pele e prematuridade segundo as associações dos descritores e os bancos de dados.

\begin{tabular}{lcccccc}
\hline \multirow{2}{*}{ Resultados da associação dos descritores } & \multicolumn{2}{c}{ PubMed } & & \multicolumn{2}{c}{ BVS } \\
\cline { 2 - 3 } "Race or ethnic group" AND "infant premature" & Geral & Período do estudo (2011-2014) & & Geral & Período do estudo (2010-2014) \\
\hline "race" AND "obstetric labor premature" & 1.480 & 481 & 661 & 166 \\
"ethnicity and health" AND "infant premature" & 421 & 68 & & 105 & 18 \\
"ethnicity and health" AND "obstetric labor premature" & 3 & 2 & 328 & 173 \\
\hline Total & 1.907 & 554 & 1.163 & 15 \\
\hline
\end{tabular}

BVS: Biblioteca Virtual em Saúde 


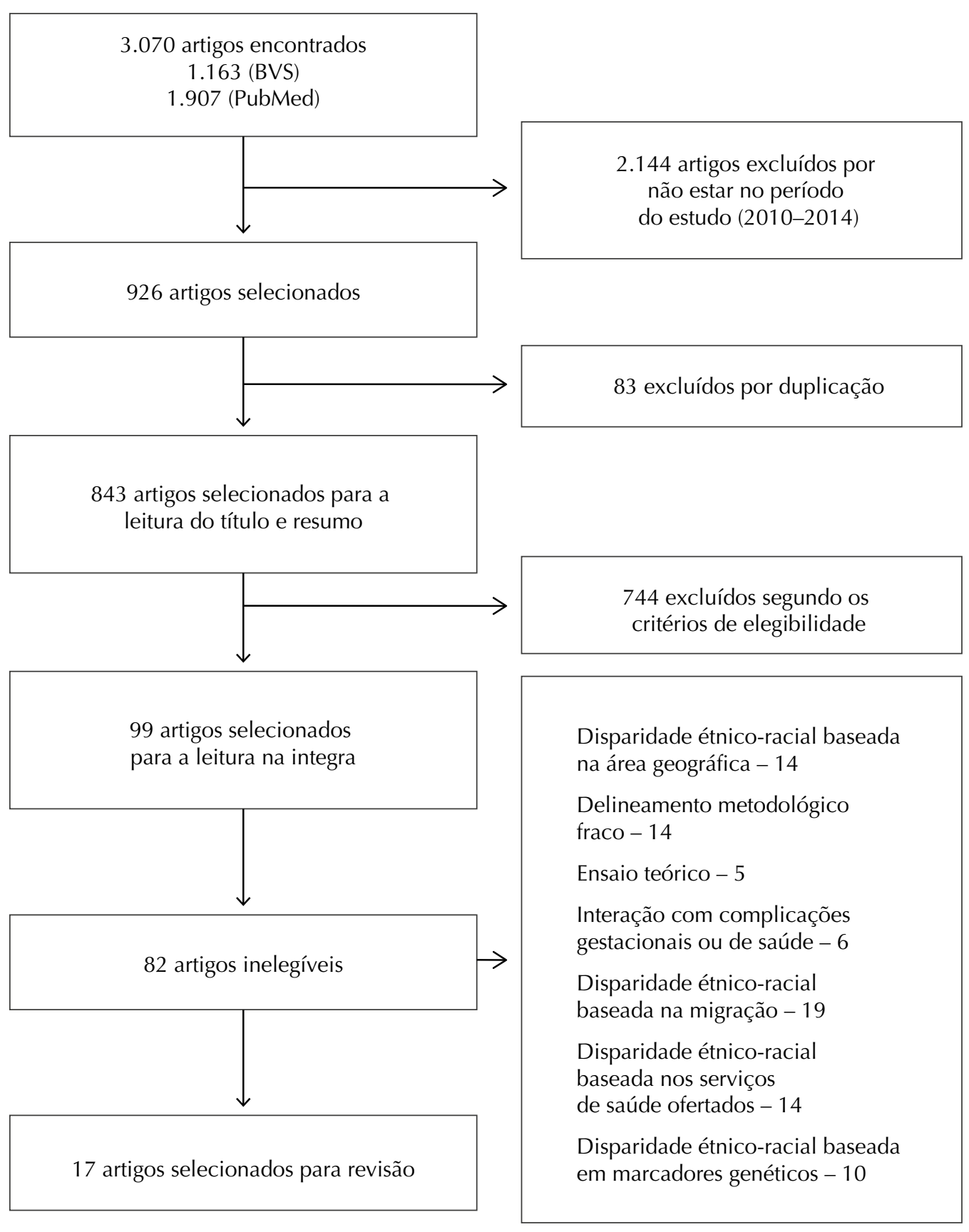

Figura 1. Fluxograma para seleção dos estudos observacionais avaliando raça/cor da pele associado à prematuridade.

\section{RESULTADOS}

A maioria dos artigos (35,3\%) foi publicada em 2013 e todos os artigos (100\%) foram realizados nos Estados Unidos. Para a classificação da idade gestacional, foi aplicada a data da última menstruação (DUM) como critério de definição em todos os estudos e a raça não negra como categoria de referência. Com exceção de um artigo ${ }^{26}$, os estudos selecionados relataram aumento do risco ou chance ajustada de parto prematuro dentro do grupo étnico/racial negro investigado quando comparados ao não negro (Tabela 2).

Os 17 estudos foram agrupados comparando o risco da ocorrência de parto prematuro entre as mulheres negras e não negras. Os artigo ${ }^{27-32}$ apontaram associação positiva entre a raça/cor da pele e a ocorrência da PMT. As mulheres negras apresentaram risco 51,0\% maior de parto prematuro, comparado com mulheres não negras ( $R R=1,51$; IC95\% 1,39-1,65). 
Tabela 2. Síntese dos artigos que avaliaram a associação entre a raça/cor da pele e a prematuridade, 2010 a 2014.

\begin{tabular}{|c|c|c|c|c|c|c|c|c|c|}
\hline Autor/Ano & $\begin{array}{l}\text { Local do } \\
\text { estudo }\end{array}$ & $\begin{array}{l}\text { Tipo de } \\
\text { estudo }\end{array}$ & $\begin{array}{l}\text { Amostra } \\
\text { (n) }\end{array}$ & Idade materna & $\begin{array}{c}\text { Prematuridade } \\
(\%)\end{array}$ & $\begin{array}{l}\text { Incidência } \\
\text { em negros }\end{array}$ & $\begin{array}{c}\text { Incidência } \\
\text { em não } \\
\text { negros }\end{array}$ & $\begin{array}{c}\text { Medida de } \\
\text { associação } \\
\text { ajustada }\end{array}$ & $\begin{array}{l}\text { Qualidade } \\
\text { do artigo }\end{array}$ \\
\hline $\begin{array}{l}\text { Almeida et al. }{ }^{27} \\
(2014)\end{array}$ & $\begin{array}{c}\text { Nova York/ } \\
\text { EUA }\end{array}$ & Transversal & 4.443 & $\begin{array}{c}80 \% \text { com menos } \\
\text { de } 35 \text { anos }\end{array}$ & 8,0 & 14,6 & 5,4 & $\mathrm{OR}=3,01$ & Bom \\
\hline $\begin{array}{l}\text { Castrillio et al. }{ }^{28} \\
(2014)\end{array}$ & Chicago/EUA & Transversal & 267.303 & Até 35 anos & 5,3 & 19,8 & 9,0 & $R R=1,2$ & Excelente \\
\hline $\begin{array}{l}\text { Collins Jr et al. }{ }^{29} \\
(2013)\end{array}$ & Chicago/EUA & Transversal & 267.303 & $\begin{array}{c}60 \% \\
(20 \text { a } 29 \text { anos })\end{array}$ & 7,2 & 15,1 & 6,7 & $\mathrm{OR}=1,9$ & Bom \\
\hline Flores et al. ${ }^{30}(2012)$ & Chicago/EUA & Transversal & 196.617 & $\begin{array}{c}86 \% \\
\text { (20 a } 34 \text { anos) }\end{array}$ & 14,0 & 9,0 & 8,1 & $\mathrm{OR}=1,04$ & Bom \\
\hline $\begin{array}{l}\text { Hwang et al. }{ }^{31} \\
(2013)\end{array}$ & $\begin{array}{l}\text { Washington e } \\
\text { Montana/EUA }\end{array}$ & $\begin{array}{c}\text { Coorte } \\
\text { retrospectiva }\end{array}$ & 24.648 & $<18$ anos & 9,6 & 11,0 & 8,0 & $\mathrm{OR}=1,34$ & Bom \\
\hline $\begin{array}{l}\text { Shempf et al. }{ }^{32} \\
(2011)\end{array}$ & $\begin{array}{l}\text { Carolina do } \\
\text { Norte/EUA }\end{array}$ & Transversal & 31.489 & $\begin{array}{c}50 \% \\
(25 \text { a } 34 \text { anos })\end{array}$ & 7,4 & 13,1 & 6,9 & $R R=3,0$ & Excelente \\
\hline Shaw et al. ${ }^{33}$ (2010) & EUA & $\begin{array}{c}\text { Coorte } \\
\text { retrospectiva }\end{array}$ & 1.223 .751 & 20 a 35 anos & 12,5 & 15.6 & 10,17 & $\mathrm{OR}=1,37$ & Bom \\
\hline Shaw et al..$^{39}(2013)$ & EUA & $\begin{array}{c}\text { Coorte } \\
\text { retrospectiva }\end{array}$ & 2.646 .176 & 20 a 29 anos & 9,9 & 15,6 & 8,5 & $\mathrm{OR}=1,09$ & Bom \\
\hline $\begin{array}{l}\text { Sullivan et al. }{ }^{34} \\
(2012)\end{array}$ & Texas/EUA & Transversal & 369.839 & $\geq 18$ anos & 13,7 & 18,4 & 12,3 & $\mathrm{OR}=0,48$ & Excelente \\
\hline $\begin{array}{l}\text { Whitehead; Helms }{ }^{40} \\
\text { (2010) }\end{array}$ & $\begin{array}{c}\text { Nova York/ } \\
\text { EUA }\end{array}$ & Transversal & 343.988 & 18 a 34 anos & 7,2 & 7,3 & 7,8 & $\mathrm{RR}=0,95$ & Bom \\
\hline $\begin{array}{l}\text { Xiong; Pridjian; } \\
\text { Dickey }^{41}(2013)\end{array}$ & EUA & $\begin{array}{c}\text { Coorte } \\
\text { retrospectiva }\end{array}$ & 50.377 & $\begin{array}{c}>70 \% \\
(30 \text { a } 39 \text { anos })\end{array}$ & 13,7 & 24,0 & 12,9 & $\mathrm{OR}=2,1$ & Regular \\
\hline $\begin{array}{l}\text { Zhang et al. }{ }^{35} \\
(2013)\end{array}$ & EUA & Transversal & 1.472 .912 & $\begin{array}{c}>50 \% \\
(18 \text { a } 24 \text { anos })\end{array}$ & 5,7 & 10,0 & 7,2 & $\mathrm{OR}=1,34$ & Regular \\
\hline $\begin{array}{l}\text { Mohamed et al. }{ }^{36} \\
(2012)\end{array}$ & EUA & $\begin{array}{c}\text { Coorte } \\
\text { retrospectiva }\end{array}$ & 17.338 & 25 a 35 anos & 10,1 & 22,1 & 12,8 & $\mathrm{OR}=1,61$ & Bom \\
\hline $\begin{array}{l}\text { Coley; Aronson }{ }^{42} \\
(2013)\end{array}$ & $\begin{array}{l}\text { Carolina do } \\
\text { Norte/EUA }\end{array}$ & Transversal & 10.515 & 17 a 19 anos & 8,4 & 11,0 & 9,2 & - & Regular \\
\hline Jongh et al. ${ }^{38}(2014)$ & EUA & $\begin{array}{l}\text { Coorte } \\
\text { retrospectivo }\end{array}$ & 11.711 & 20 a 34 anos & 3,9 & 13,5 & 8,8 & $\mathrm{OR}=1,36$ & Bom \\
\hline $\begin{array}{l}\text { Fujimoto et al. }{ }^{37} \\
(2010)\end{array}$ & EUA & $\begin{array}{l}\text { Coorte } \\
\text { retrospectivo }\end{array}$ & 139,027 & 35 a 39 anos & 15,2 & 21,0 & 14,8 & $\mathrm{OR}=1,79$ & Excelente \\
\hline $\begin{array}{l}\text { Torloni et al. }{ }^{26} \\
(2012)\end{array}$ & $\begin{array}{c}\text { Tennessee/ } \\
\text { EUA }\end{array}$ & $\begin{array}{c}\text { Caso } \\
\text { controle }\end{array}$ & 1.762 & 20 a 34 anos & 8,3 & 21,7 & 27,6 & $\mathrm{OR}=1,29$ & Bom \\
\hline
\end{tabular}

Identificação dos Estudos

RR (IC95\%)

$\%$

Almeida et al. ${ }^{27}(2014)$
Castrillio et al. ${ }^{28}(2014)$
Collins Jr et al. ${ }^{.9}(2013)$
Flores et al. ${ }^{30}(2012)$
Hwang et al. ${ }^{31}(2013)$
Shempf et al. ${ }^{32}(2011)$
Shaw et al. ${ }^{33}(2010)$
Shaw et al. ${ }^{39}(2013)$
Sullivan et al. ${ }^{34}(2012)$
Whitehead; Helms ${ }^{40}(2010)$
Xiong; Pridjian; Dickey ${ }^{41}(2013)$
Zhang et al. ${ }^{35}(2013)$
Mohamed et al. ${ }^{36}(2012)$
Coley; Aronson ${ }^{42}(2013)$
Jongh et al. ${ }^{38}(2014)$
Fujimoto et al. ${ }^{37}(2010)$
Torloni et al. ${ }^{26}(2012)$
Overall (l-squared $=99,6 \%, p=0,000)$

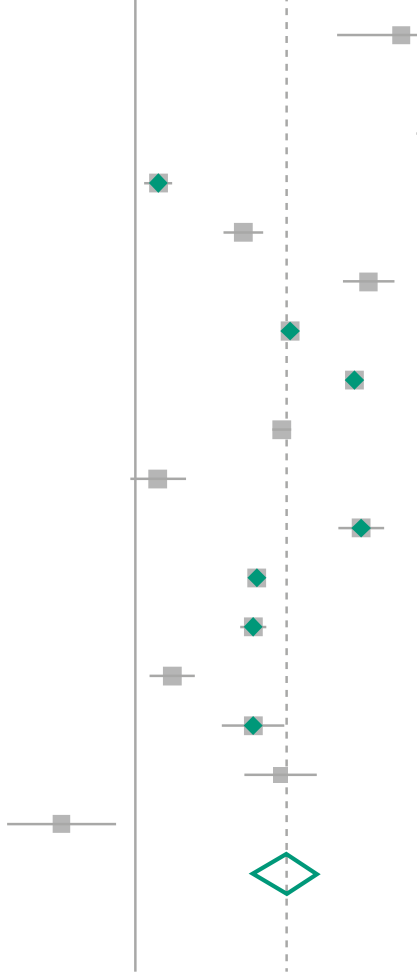

$2,88(2,77-3,00)$

$1,07(1,02-1,11)$

$1,35(1,27-1,42)$

$1,90(1,77-2,04)$

$1,53(1,52-1,55)$

$1,83(1,81-1,84)$

$1,50(1,46-1,54)$

$1,06(0,99-1,15)$

$1,86(1,75-1,98)$

$1,40(1,38-1,41)$

$1,38(1,34-1,43)$

$1,11(1,04-1,18)$

$1,38(1,27-1,51)$

$1,49(1,35-1,65)$

$0,82(0,70-0,95)$

5,2

$1,51(1,39-1,65)$

100,0

Nota: Os valores dos artigos foram obtidos por meio de análise de efeitos aleatórios.

Figura 2. Forest plot dos estudos observacionais sobre associação da raça/cor da pele e prematuridade. 


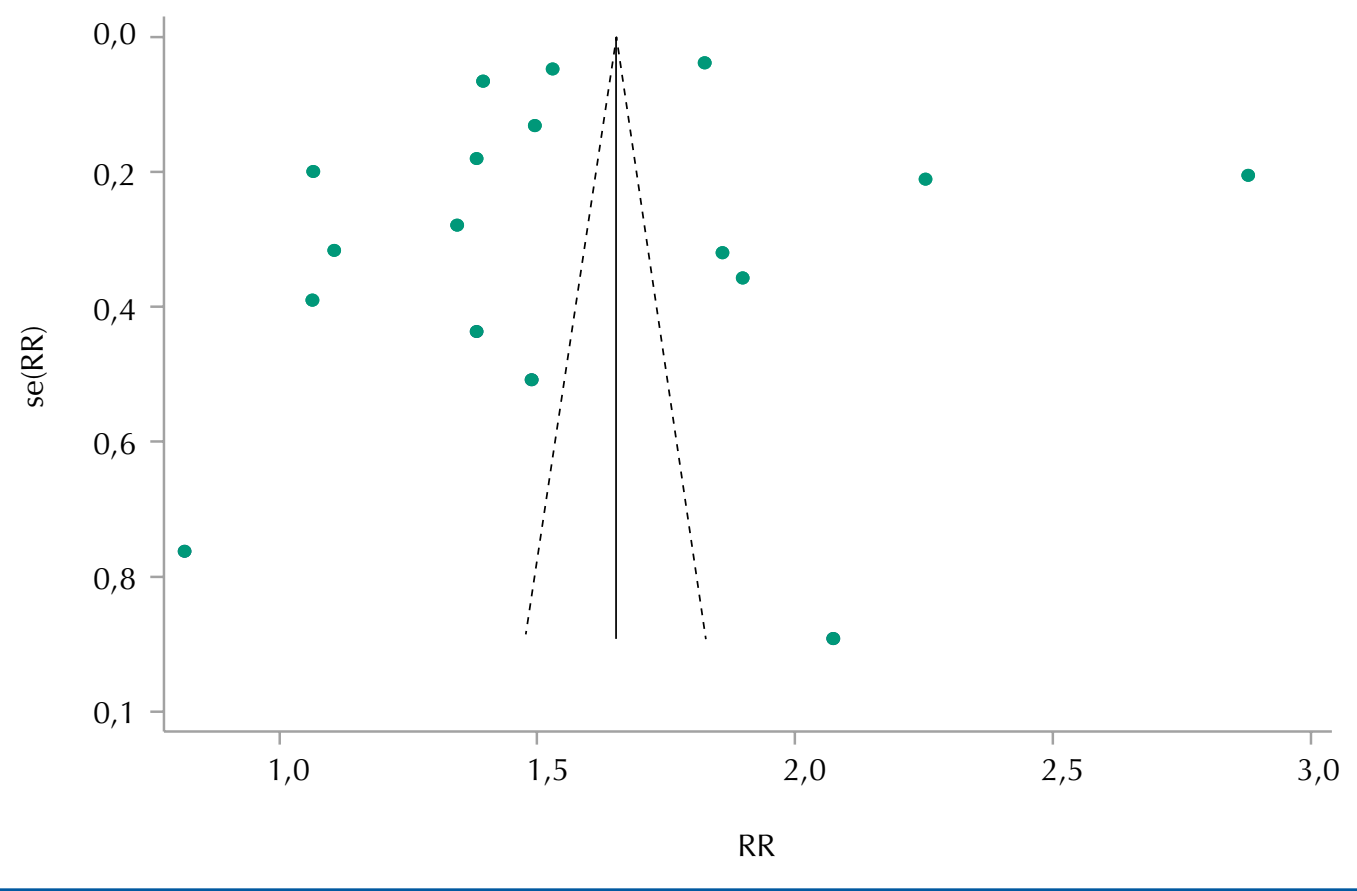

Figura 3. Gráfico de funil da associação entre raça/cor da pele e prematuridade.

O resultado do teste de inconsistência mostrou elevada heterogeneidade entre os estudos analisados (99,6\%; p = 0,00). Assim, o modelo de efeitos aleatórios foi utilizado para calcular a medição de síntese (Figura 2).

Os resultados mostraram grande variabilidade entre os estudos, o que denotou a presença de viés de publicação (Figura 3). Cinco estudos maiores apareceram na parte superior do gráfico, 10 estudos com amostras médias localizados na parte mais central à esquerda do gráfico e dois estudos na parte inferior, pois tinham amostras menores. Todos os estudos se apresentaram fora do slope do gráfico.

\section{DISCUSSÃO}

Os resultados da presente meta-análise confirmam a associação entre a raça/cor da pele e a ocorrência de PMT: mulheres negras tiveram uma vez e meia mais chance de ter parto prematuro, quando comparadas com mulheres não negras. Esse resultado sustenta a hipótese de que a raça/cor da pele é um fator de risco para a prematuridade e corrobora os achados de publicações prévias de estudos observacionais e revisões sistemáticas ${ }^{7,16,33}$.

A associação entre a raça/cor da pele e a PMT não tem seu mecanismo totalmente esclarecido na perspectiva da determinação social, tomada como marco teórico neste estudo. Entretanto, a literatura faz referência a fatores maternos que interferem na prematuridade como a altura e o peso da mãe, paridade e complicações durante a gravidez, mães adolescentes (especialmente se < 15 anos). São citados também os fatores comportamentais que influenciam a PMT, incluindo tabagismo, consumo de álcool e drogas durante a gravidez e a assistência pré-natal. A condição socioeconômica, mensurada pela renda familiar, ter habitação, a ocupação, a educação, o tipo do trabalho materno e a maternidade solitária, também influencia na PMT. Todos esses fatores de risco se interseccionam para o nascimento prematuro e estão mais fortemente presentes na vida das mulheres negras devido à posição de desvantagem que a elas é destinada pela sociedade. Mulheres negras geralmente têm pior situação socioeconômica e pior condição de ter uma boa nutrição. Além disso, estão expostas a sofrer discriminação por sua identidade étnica racial e esse estresse psicológico também pode levá-las a ter filhos prematuros ${ }^{1,34-36}$. 
Acredita-se que a interação entre fatores genéticos e ambientais influenciem a raça/cor da pele como umas das causas para a ocorrência da $\mathrm{PMT}^{37}$. Nesse sentido, as desigualdades étnico-raciais podem ser visualizadas no acesso aos serviços de saúde, às oportunidades socioeconômicas e aos fatores genéticos. Constitui assim, uma situação desigual enfrentada pelas mulheres negras ${ }^{37}$.

Os estudos incluídos foram pontuados como bom ou excelente após avaliação da qualidade. Pode-se perceber grande variação no tamanho da amostra dos estudos incluídos. O estudo de Torloni et al. ${ }^{26}$ apresentou 1.762 mulheres e o estudo de Shaw et al..$^{38}$ apresentou 2.646 .176 mulheres. No entanto, o tamanho da amostra apresentou pouco impacto sobre a generalização dos resultados.

Apenas no estudo de Hwang et al. ${ }^{31}$ a maioria das participantes tinha idade inferior a 18 anos. Como houve ajuste dessa covariável neste estudo, acredita-se que ela não influenciou nos resultados desta meta-análise. Do mesmo modo, as variáveis que poderiam estar associadas com a ocorrência da PMT, como fatores socioeconômicos e comportamentais, também não influenciaram a medida meta-analítica no presente estudo, apesar da alta heterogeneidade identificada. Isso indica a variação entre os resultados dos estudos analisados.

Para todos os estudos incluídos, a informação sobre a idade gestacional foi obtida de um registro hospitalar ou do serviço de saúde e a DUM foi adotada como critério de definição para a idade gestacional. Esse procedimento reduziu a possibilidade de ocorrência de viés de memória.

Os estudos incluídos apresentaram grande variação na ocorrência da PMT. As taxas de prematuridade em mulheres negras variaram de $7,3 \%^{39}$ a $24,0 \%{ }^{40} \mathrm{e}$ essa variação é ainda maior entre as mulheres não negras, que foi de $6,7^{41}$ a 27,6 $6^{42}$.Já para a medida ajustada da associação entre a raça/cor e a PMT, a maior prevalência da PMT foi de $8,0 \%{ }^{27}$. Os autores mostraram que houve diferenças significativas por raça/cor em todas as variáveis sociodemográficas, comportamentais e médicas, exceto para história pregressa de parto prematuro.

Mulheres negras tiveram maiores chances de parto prematuro quando comparadas às mulheres não negras. Resultados semelhantes foram encontrados no estudo realizado no Reino Unido, em que as mulheres negras tiveram 1,5 vezes mais chances de ter partos prematuros ${ }^{19}$. Esses achados expressam a dificuldade de acesso aos serviços de saúde por mulheres e crianças de diferentes grupos étnicos. Isso pode explicar as diferenças encontradas nos níveis de ocorrência da PMT devido à ausência de prevenção para riscos evitáveis a partir de assistência integral.

Em relação ao tamanho da amostra, o estudo de Torloni et al..$^{26}$ apresentou menor número de participantes, o que pode ser explicado pelo método utilizado, que é de caso-controle. O estudo apresentou prevalência de $35,4 \%$ para a ocorrência de parto prematuro na população total do estudo. No entanto, foi evidenciada uma prevalência maior entre as mulheres não negras quando comparadas às mulheres negras, o que difere da maioria dos estudos selecionados. Uma das limitações deste estudo foi o pequeno número de participantes, o que pode ter dificultado a análise sobre os efeitos da PMT em mulheres de diferentes origens étnicas.

O estudo de Xiong et al. ${ }^{41}$ mostrou a ocorrência do parto prematuro em mulheres negras de 4,8 (IC95\% 4,1-5,7), quando comparadas às mulheres brancas e apresentou prevalência de 13,7\% para a população do estudo. Uma das principais limitações do estudo foi em relação à informação sobre a raça/cor. Cerca de 35,0\% estavam faltantes e em relação ao controle dos fatores de confundimento, pois foi frequente a ausência de informações sobre os dados obstétricos e sobre as condições clínicas pré-existentes. Entretanto, o estudo tem como ponto forte o tamanho da amostra, suficiente para analisar a associação entre a raça/cor e a prematuridade.

Para a maior parte dos estudos, a informação sobre o peso ao nascer foi obtida de um registro hospitalar, reduzindo a possibilidade de ocorrência de viés de memória. No entanto, o número de artigos incluídos nesta meta-análise foi pequeno em relação ao número identificado na revisão sistemática da literatura. Como resultado, há um potencial viés de seleção. 
A maioria dos estudos incluídos nesta meta-análise foi de corte transversal, limitando a capacidade de distinguir relações temporais subjacentes à associação entre a raça/cor da pele e a ocorrência da PMT. No entanto, esta é uma limitação dos estudos publicados atualmente neste campo. Como esta é uma meta-análise de estudos observacionais, este estudo enfrentou o desafio inerente de resumir os resultados dos estudos com diferentes delineamentos epidemiológicos.

Os resultados da presente meta-análise evidenciaram a associação entre a raça/cor da pele e a PMT. Mulheres negras têm o risco 1,51 vezes maior de ter um parto prematuro do que as mulheres brancas. Esse achado pode subsidiar tomadas de decisões e fornecer elementos que fundamentem os esforços para atingir a equidade em saúde.

Entretanto, o pertencimento por raça/cor da pele e a PMT apresentam aspectos sociais, comportamentais e biológicos que são complexos. Estudos futuros poderão investigar a associação da raça/cor da pele com a PMT considerando estes aspectos, principalmente no tocante às mulheres negras por sua inserção social e econômica desfavorável. Situação esta agravada pelo tratamento que a sociedade dá a determinados grupos ao considerar o seu pertencimento étnico racial.

\section{REFERÊNCIAS}

1. Goldenberg RL, Culhane JF, lams JD, Romero R. Epidemiology and causes of preterm birth. Lancet. 2008;371(9606):75-84. https://doi.org/10.1016/S0140-6736(08)60074-4

2. Dória MT, Spautz CC. Trabalho de parto prematuro: predição e prevenção. Femina. 2011 [citado 19 dez 2015];39:443-9. Disponível em: http://files.bvs.br/upload/S/0100-7254/2011/v39n9/a2957.pdf

3. Beck S, Wojdyla D, Say L, Betran AP, Merialdi M, Requejo JH, et al. The worldwide incidence of preterm birth: a systematic review of maternal mortality and morbidity. Bull Word Health Organ. 2010;88(1):31-8. https://doi.org/10.2471/BLT.08.062554

4. Bettiol HS, Barbieri MA, Silva AAM. Epidemiologia do nascimento pré-termo: tendências atuais [editorial]. Rev Bras Ginecol Obstet. 2010;32(2):57-60. https://doi.org/10.1590/S0100-72032010000200001

5. Silveira MF, Santos IS, Barros AJD, Matijasevich A, Barros FC, Victora CG. Aumento da prematuridade no Brasil: revisão de estudos de base populacional. Rev Saude Publica. 2008;42(5):957-64. https://doi.org/10.1590/S0034-89102008000500023

6. Matijasevich A, Silveira MF, Matos ACG, Rabello Neto D, Fernandes RM, Maranhão AG, et al. Estimativas corrigidas da prevalência de nascimentos pré-termo no Brasil, 2000 a 2001. Epidemiol Serv Saude. 2013;22(4):557-64. https://doi.org/10.5123/S1679-49742013000400002

7. Kramer MR, Hogue CJ, Dunlop AL, Menon R. Preconceptional stress and racial disparities in preterm birth: an overview. Acta Obstet Gynecol Scand. 2011;90(12):1307-16. https://doi.org/10.1111/j.1600-0412.2011.01136.x

8. Pearce N, Foliaki S, Sporle A, Cunningham C. Genetics, race, ethnicity and health. BMJ. 2004;328(7447):1070-2. https://doi.org/10.1136/bmj.328.7447.1070

9. Cooper R. A note on the biologic concept of race and its application in epidemiologic research. Am Heart J. 1984;108(3 Pt 2):715-22. https://doi.org/10.1016/0002-8703(84)90662-8

10. Dressler WW, Oths KS, Gravlee CC. Race and ethnicity in Public Health research: models to explain health disparities. Annu Rev Anthropol. 2005;34(1):231-52. https://doi.org/10.1146/annurev.anthro.34.081804.120505

11. Evans RG. Introduction. In: Evans RG, Barer ML, Marmor TR, organizers. Why are some people healthy and others not? The determinants of health of populations. New York: Aldine de Gruyter; 1994. p.3-26. (Social Institutions and Social Change)

12. Goodman AH. Why genes don't count (for racial differences in health). Am J Public Health. 2000;90(11):1699-702. https://doi.org/10.2105/AJPH.90.11.1699

13. Lovell PA, Wood CH. Skin color, racial identity, and life chances in Brazil. Lat Am Perspect. 1998;25(3):90-109. https://doi.org/10.1177/0094582X9802500305 
14. Krieger N. Discrimination and health. In: Berkman LF, Kawachi I, editors. Social epidemiology. New York: Oxford University Press; 2000. p.36-75.

15. Wilkinson R, Marmot MG. Social determinants of health: the solid facts. 2.ed. Copenhagen: World Health Organization Regional Office for Europe; 2003 [citado 19 dez 2015]. Disponível em: http://www.euro.who.int/_data/assets/pdf_file/0005/98438/e81384.pdf

16. Menon R, Dunlop AL, Kramer MR, Fortunato SJ, Hogue CJ. An overview of racial disparities in preterm birth rates: caused by infection or inflammatory response? Acta Obstet Gynecol Scand. 2011;90(12):1325-31. https://doi.org/10.1111/j.1600-0412.2011.01135.x

17. Vianna M. Parto prematuro: prevenção. Femina. 2006;34(11):731-4.

18. Jongh BE, Locke R, Paul DA, Hoffman M. The differential effects of maternal age, race/ethnicity and insurance on neonatal intensive care unit admission rates. BMC Pregnancy Childbirth. 2012;12:97. https://doi.org/10.1186/1471-2393-12-97

19. Patel RR, Steer P, Doyle P, Little M, Elliot P. Does gestation vary by ethnic group? A London-based study of over 122,000 pregnancies with spontaneous onset of labour. Int J Epidemiol. 2004;33(1):107-13. https://doi.org/10.1093/ije/dyg238

20. Perpétuo IHO. Raça e acesso ás ações prioritárias na agenda da saúde reprodutiva. J Rede Fem Saude. 2000;22:10-6.

21. Chacham A. Cesárea e esterilização: condicionantes socioeconômicos, etários e raciais. J Rede Saude. 2001;23:44-7.

22. Silva LM, Silva RA, Silva AAM, Bettiol H, Barbieri MA. Racial inequalities and perinatal health in the southeast region of Brazil. Braz J Med Biol Res. 2007;40(9):1187-94. https://doi.org/10.1590/S0100-879X2006005000144

23. Urrútia G, Bonfill X. Declaración PRISMA: una propuesta para mejorar la publicación de revisiones sistemáticas y metaanálises. Med Clin (Barc). 2010 [citado 19 dez 2015];135(11):507-11. Disponível em: http://www.laalamedilla.org/Investigacion/Recursos/ PRISMA\%20Spanish\%20Sept\%202010.pdf

24. Schaaf JM, Liem SM, Mol BW, Abu-Hanna A, Ravelli AC. Ethnic and racial disparities in the risk of preterm birth: a systematic review and meta-analysis. Am J Perinatol. 2013;30(6):433-50. https://doi.org/10.1055/s-0032-1326988

25. Wells GA, Shea B, O'Connell D, Peterson J, Welch V, Losos M, et al. The NewcastleOttawa Scale (NOS) for assessing the quality of nonrandomized studies in meta-analyses. Ottawa: Ottawa Hospital Research Institute; 2014 [citado 19 dez 2015] Disponível em: http://www.ohri.ca/programs/clinical_epidemiology/nos_manual.pdf

26. Torloni MR, Fortunato SJ, Betrán AP, Williams S, Brou L, Drobek CO, et al. Ethnic disparity in spontaneous preterm birth and maternal pre-pregnancy body mass index. Arch Gynecol Obstet. 2012;285(4):959-66. https://doi.org/10.1007/s00404-011-2102-8

27. Almeida J, Mulready-Ward C, Bettegowda VR, Ahluwalia IB. Racial/ethnic and nativity differences in birth outcomes among mothers in New York City: the role of social ties and social support. Matern Child Health J. 2014;18(1):90-100. https://doi.org/10.1007/s10995-013-1238-5

28. Castrillio SM, Rankin KM, David RJ, Collins JW Jr. Small-for-gestational age and preterm birth across generations: a population-based study of Illinois births. Matern Child Health J. 2014;18(10):2456-64. https://doi.org/10.1007/s10995-014-1484-1

29. Collins JW Jr, Rankin KM, Janowiak CM. Suburban migration and the birth outcome of Chicago-born white and African-American women: the merit of the healthy migrant theory? Matern Child Health J. 2013;17(9):1559-66. https://doi.org/10.1007/s10995-012-1154-0

30. Flores ME, Simonsen SE, Manuck TA, Dyer JM, Turok DK. The "Latina epidemiologic paradox": contrasting patterns of adverse birth outcomes in U.S.-born and foreign-born Latinas. Womens Health Issues. 2012;22(5):e501-7. https://doi.org/10.1016/j.whi.2012.07.005

31. Hwang M, Shrestha A, Yazzie S, Jackson ML. Preterm birth among American Indian/Alaskan natives in Washington and Montana: comparison with non-Hispanic Whites. Matern Child Health J. 2013;17(10):1908-12. https://doi.org/10.1007/s10995-012-1215-4

32. Schempf AH, Kaufman JS, Messer LC, Mendola P. The neighborhood contribution to black-white perinatal disparities: an example from two North Carolina counties, 1999-2001. Am J Epidemiol. 2011;174(6):744-52. https://doi.org/10.1093/aje/kwr128 
33. Shaw RJ, Pickett KE, Wilkinson RG. Ethnic density effects on birth outcomes and maternal smoking during pregnancy in the US linked birth and infant death data set. Am J Public Health. 2010;100(4):707-13. https://doi.org/h10.2105/AJPH.2009.167114

34. Sullivan K, Raley RK, Hummer RA, Schiefelbein E. The potential contribution of maritalcohabitation status to racial, ethnic, and nativity differentials in birth outcomes in Texas. Matern Child Health J. 2012;16(4):775-84. https://doi.org/10.1007/s10995-011-0801-1

35. Zhang S, Cardarelli K, Shim R, Ye J, Booker KL, Rust G. Racial disparities in economic and clinical outcomes of pregnancy among Medicaid recipients. Matern Child Health J. 2013;17(8):1518-25. https://doi.org/10.1007/s10995-012-1162-0

36. Mohamed MA, Ahmad T, Macri C, Aly H. Racial disparities in maternal hemoglobin concentrations and pregnancy outcomes. J Perinat Med. 2012;40(2):141-9. https://doi.org/10.1515/jpm.2011.137

37. Fujimoto VY, Luke B, Brown MB, Jain T, Armstrong A, Grainger DA, et al. Racial and ethnic disparities in assisted reproductive technology outcomes in the United States. Fertil Steril. 2010;93(2):382-90. https://doi.org/10.1016/j.fertnstert.2008.10.061

38. Jongh BE, Paul DA, Hoffman M, Locke R. Effects of pre-pregnancy obesity, race/ethnicity and prematurity. Matern Child Health J. 2014;18(3):511-7. https://doi.org/10.1007/s10995-013-1296-8

39. Shaw RJ, Pickett KE. The health benefits of Hispanic communities for non-Hispanic mothers and infants: another Hispanic paradox. Am J Public Health. 2013;103(6):1052-7. https://doi.org/10.2105/AJPH.2012.300985

40. Whitehead N, Helms K. Racial and ethnic differences in preterm delivery among low-risk women. Ethn Dis. 2010 [citado 19 dez 2015];20(3):261-6. Disponível em: https://www.ethndis.org/priorarchives/ethn-20-03-261.pdf

41. Xiong X, Pridjian G, Dickey RP. Racial and ethnic disparities in preterm births in infants conceived by in vitro fertilization in the United States. Am J Obstet Gynecol. 2013;209(2):128.e1-128.e6. https://doi.org/10.1016/j.ajog.2013.04.012

42. Coley SL, Aronson RE. Exploring birth outcome disparities and the impact of prenatal care utilization among North Carolina teen mothers. Womens Health Issues. 2013;23(5):e287-94. https://doi.org/10.1016/j.whi.2013.06.004

Contribuição dos Autores: Concepção e planejamento do estudo: KAO, DBS, EMA. Coleta, análise e interpretação dos dados: KAO, KAO, DBS. Elaboração ou revisão do manuscrito: KAO, DBS, EMA, CAC, CALS. Aprovação da versão final: KAO, DBS, EMA. Responsabilidade pública pelo conteúdo do artigo: KAO, DBS, EMA.

Conflito de Interesses: Os autores declaram não haver conflito de interesses. 